\title{
On Some Partition Functions Related to Some Mock Theta Functions
}

by

\author{
Alexander E. PATKOWSKI \\ Presented by Jerzy KACZOROWSKI
}

Summary. We show that some partitions related to two of Ramanujan's mock theta functions are related to indefinite quadratic forms and real quadratic fields. In particular, we examine a third order mock theta function and a fifth order mock theta function.

1. Introduction. In [2, Theorem 6], Andrews, Dyson, and Hickerson proved the following $q$-series identity:

$$
\begin{aligned}
\sum_{n=1}^{\infty} \frac{(-1)^{n} q^{n^{2}}}{(1-q)\left(1-q^{3}\right) \cdots} & \left(1-q^{2 n-1}\right) \\
= & \sum_{n=1}^{\infty}(-1)^{n} q^{n(3 n-1)}\left(1+q^{2 n}\right) \sum_{j=0}^{2 n-1} q^{-j(j+1) / 2} .
\end{aligned}
$$

This was rather surprising because they were able to show that certain partitions related to those enumerated by one of Ramanujan's third order mock theta functions can be represented by indefinite quadratic forms. Let $\beta(n)$ be the number of partitions of $n$ into odd parts, with the property that if $k$ occurs as a part, then all positive odd numbers less than $k$ also occur. If $S_{3}(n)$ (resp. $S_{1}(n)$ ) denotes the number of partitions counted by $\beta(n)$ with largest part $\equiv 3(\bmod 4)(\operatorname{resp} . \equiv 1(\bmod 4))$ then

$$
\sum_{n=1}^{\infty}\left(S_{3}(n)-S_{1}(n)\right) q^{n}=\sum_{n=1}^{\infty} \frac{(-1)^{n} q^{n^{2}}}{(1-q)\left(1-q^{3}\right) \cdots\left(1-q^{2 n-1}\right)}
$$

Interestingly, this is the same partition function generated by a third order mock theta function (see Section 3 below), but with an additional 
weight function attached. In this paper, we establish some further information about the partition functions $S_{3}(n)$ and $S_{1}(n)$, by establishing a new $q$-series identity that is closely related to (1). Further, we relate the difference of $S_{1}(n)$ and $S_{3}(n)$ to the arithmetic of $\mathbb{Q}(\sqrt{3})$. We also show that the partition function enumerated by Ramanujan's fifth order mock theta function [1]

$$
\sum_{n=0}^{\infty}(-q)_{n} q^{(n+1)(n+2) / 2},
$$

with an additional weight function, is related to the arithmetic of $\mathbb{Q}(\sqrt{15})$.

TheOREM 1.1. For $n \equiv 1(\bmod 24)$, let $s_{1}(n)$ be the excess of the number of inequivalent solutions to $x^{2}-48 y^{2}=n$ for which $x+6 y \equiv \pm 1(\bmod 12)$ over the number of those for which $x+6 y \equiv \pm 5(\bmod 12)$. Then

$$
2(-1)^{n}\left(S_{3}(n)-S_{1}(n)\right)=s_{1}(1-24 n) .
$$

From here we can relate this partition function to the arithmetic of $\mathbb{Q}(\sqrt{3})$. The proof of the next result is straightforward, but rather tedious, and so we leave this for the reader.

COROllaRY 1.2. For $n \equiv 1(\bmod 24)$, let $s_{2}(n)$ be the excess of the number of inequivalent solutions to $x^{2}-3 y^{2}=n$ for which either $x \equiv \pm 2$ $(\bmod 24)$ or $2 x+3 y \equiv \pm 2(\bmod 24)$ over the number of those for which either $x \equiv \pm 10(\bmod 24)$ or $2 x+3 y \equiv \pm 10(\bmod 24)$. Then

$$
2(-1)^{n}\left(S_{3}(n)-S_{1}(n)\right)=s_{2}(1-24 n) .
$$

Our next result is slightly more elegant, and is mentioned in the acknowledgments. In particular, we state a result mentioned in [2, Section 6] involving a relation of $S_{3}(n)-S_{1}(n)$ to the arithmetic of $\mathbb{Q}(\sqrt{3})$.

Corollary 1.3. For $n \equiv 1(\bmod 24)$, let $I(n)$ be the excess of the number of inequivalent solutions to $x^{2}-3 y^{2}=n$ for which $x+3 y \equiv \pm 1$ or $\pm 5(\bmod 24)$ over the number of those for which $x+3 y \equiv \pm 7$ or \pm 11 $(\bmod 24)$. Then

$$
2\left(S_{3}(n)-S_{1}(n)\right)=I(1-24 n) .
$$

THEOREM 1.4. Let $L(n)$ be the number of partitions of $n$ such that each part occurs at most twice, the largest part is unique, and if $k$ occurs as a part then all smaller positive integers occur. Let $L_{e}(n)\left(\right.$ resp. $\left.L_{o}(n)\right)$ be the number of partitions counted by $L(n)$ with an even (resp. odd) number of parts. Then $L_{o}(n)-L_{e}(n)$ equals the sum of $(-1)^{r+j}$ over all pairs $(r, j)$ such that $n=r(5 r \pm 1) / 2-j(3 j+1) / 2,-r \leq j \leq r-1$.

TheOREM 1.5. Let $f(n)$ be the excess of the number of inequivalent solutions to $x^{2}-15 y^{2}=6(60 n-1)$ for which $x+5 y \equiv \pm 2$ or $\pm 8(\bmod 60)$ 
over the number of those for which $x+5 y \equiv \pm 22$ or $\pm 28(\bmod 60)$. Then

$$
2\left(L_{o}(n)-L_{e}(n)\right)=f(n) .
$$

2. Proofs of main theorems. First we need to present some information about Bailey pairs to establish our $q$-series identities. In [4], we find the following well-known lemma that is key in our proofs of the main theorems.

BAILEY's Lemma. If , for every $n \geq 0$,

$$
\beta_{n}=\sum_{r=0}^{n} \frac{\alpha_{r}}{(q)_{n-r}(a q)_{n+r}},
$$

then

$$
\sum_{n=0}^{\infty}(x)_{n}(y)_{n}(a q / x y)^{n} \beta_{n}=\frac{(a q / x)_{\infty}(a q / y)_{\infty}}{(a q)_{\infty}(a q / x y)_{\infty}} \sum_{n=0}^{\infty} \frac{(x)_{n}(y)_{n}(a q / x y)^{n} \alpha_{n}}{(a q / x)_{n}(a q / y)_{n}} .
$$

We have employed standard notation $[6]$

$$
(a ; q)_{n}=(a)_{n}:=(1-a)(1-a q) \cdots\left(1-a q^{n-1}\right) .
$$

We say that a pair of sequences $\left(\alpha_{n}, \beta_{n}\right)$ is a Bailey pair with respect to $a$ if (3) holds.

Proof of Theorem 1.1. It is known ([1, Theorem 4] with $a=1$ and $b \rightarrow 0)$ that $\left(\alpha_{n}, \beta_{n}\right)$ is a Bailey pair with respect to 1 where

$$
\begin{aligned}
& \alpha_{n}=\left\{\begin{array}{cc}
q^{n^{2}}\left((-1)^{n} q^{-n(n-1) / 2}\left(1+q^{n}\right)\right. & \\
\left.-q^{n^{2}-n}\left(1-q^{2 n}\right) \sum_{j=-n+1}^{n-1}(-1)^{j} q^{-j(3 j+1) / 2}\right), & n>0, \\
1, & n=0,
\end{array}\right. \\
& \beta_{n}=1 .
\end{aligned}
$$

Now inserting this pair into (4) with $a=1$, and $y=-1$, we get an equation of the form (using $\beta_{n}=1$ )

$$
\begin{aligned}
1+ & (1-x) \sum_{n=1}^{\infty}(q x)_{n-1}(-1)_{n}(-q / x)^{n} \\
& =\frac{(q / x)_{\infty}(-q)_{\infty}}{(q)_{\infty}(-q / x)_{\infty}}\left(1+(1-x) \sum_{n=1}^{\infty} \frac{(x q)_{n-1}(-1)_{n}(-q / x)^{n} \alpha_{n}}{(q / x)_{n}(-q)_{n}}\right) .
\end{aligned}
$$

Now differentiating the right side of (5) with respect to $x$ and setting $x=1$ gives

$$
\sum_{n=1}^{\infty} \frac{q^{n}}{1-q^{n}}+\sum_{n=1}^{\infty} \frac{q^{n}}{1+q^{n}}-2 \sum_{n=1}^{\infty} \frac{(-q)^{n} \alpha_{n}}{1-q^{2 n}}
$$


This easily follows from the fact that $\lim _{x \rightarrow 1} \frac{d}{d x}(1-x) f(x)=-f(1)$ if $f(x)$ is differentiable at $x=1$, and the fact that

$$
\frac{d}{d x} \frac{(q / x)_{\infty}(-q)_{\infty}}{(q)_{\infty}(-q / x)_{\infty}}=\frac{(q / x)_{\infty}(-q)_{\infty}}{(q)_{\infty}(-q / x)_{\infty}}\left(\sum_{n=1}^{\infty} \frac{q^{n} / x^{2}}{1-q^{n} / x}+\sum_{n=1}^{\infty} \frac{q^{n} / x^{2}}{1+q^{n} / x}\right) .
$$

Using the identity

$$
\sum_{n=1}^{\infty} \frac{q^{n}}{1-q^{n}}+\sum_{n=1}^{\infty} \frac{q^{n}}{1+q^{n}}=2 \sum_{n=1}^{\infty} \frac{q^{2 n-1}}{1-q^{2 n-1}}
$$

gives us

$$
\begin{aligned}
-\sum_{n=1}^{\infty}\left(q^{2} ; q^{2}\right)_{n-1}(-q)^{n}= & \sum_{n=1}^{\infty} \frac{q^{2 n-1}}{\left(1-q^{2 n-1}\right)}-\sum_{n=1}^{\infty} \frac{q^{n(n+3) / 2}}{\left(1-q^{n}\right)} \\
& +\sum_{n=1}^{\infty}(-1)^{n} q^{2 n^{2}} \sum_{j=-n+1}^{n-1}(-1)^{j} q^{-j(3 j+1) / 2} .
\end{aligned}
$$

By [3] and [5], we can write the above equation as

$$
\sum_{n=1}^{\infty} \frac{q^{n^{2}}}{\left(-q ; q^{2}\right)_{n}}=\sum_{n=1}^{\infty} q^{n(n+1) / 2}+\sum_{n=1}^{\infty}(-1)^{n} q^{2 n^{2}} \sum_{j=-n+1}^{n-1}(-1)^{j} q^{-j(3 j+1) / 2} .
$$

Inspecting the sum on $j$ gives

$$
\sum_{n=1}^{\infty} \frac{q^{n^{2}}}{\left(-q ; q^{2}\right)_{n}}=\sum_{n=1}^{\infty}(-1)^{n} q^{2 n^{2}} \sum_{j=-n}^{n-1}(-1)^{j} q^{-j(3 j+1) / 2} .
$$

By (2), it is clear that the sum on the left hand side is the generating function for $(-1)^{n}\left(S_{3}(n)-S_{1}(n)\right)$. We are now ready to consider the sum on the right side of $(7)$.

The equation $n=2 r^{2}-j(3 j+1) / 2$ is equivalent to $1-24 n=$ $(6 j+1)^{2}-48 r^{2}$. So taking the weight of this sum into consideration, we find that $(-1)^{n}\left(S_{3}(n)-S_{1}(n)\right)$ equals the number of solutions of $1-24 n=$ $x^{2}-48 y^{2}$ with $x \equiv 1(\bmod 6)$ and $-6 y<x<6 y$ for which $x+6 y \equiv 1$ $(\bmod 12)$ minus the number of those for which $x+6 y \equiv-5(\bmod 12)$. Mapping $(x, y)$ to $(-x, y)$ shows that it also equals the number of solutions with $x \equiv-1(\bmod 6)$ and $-6 y<x<6 y$ for which $x+6 y \equiv-1(\bmod 12) \operatorname{minus}$ the number of those for which $x+6 y \equiv 5(\bmod 12)$. Furthermore, Lemma 3 of [2] with $D=48, x_{1}=7$, and $y_{1}=1$ shows that each equivalence class of solutions has a unique element $(x, y)$ with $-6 y<x<6 y$. The result follows after equating the coefficients of $q^{n}$ on both sides of (7).

The proofs of Corollaries 1.2 and 1.3 follow in a similar manner from the fact that the equation $n=2 r^{2}-j(3 j+1) / 2$ is equivalent to $1-24 n=$ $(6 j+1)^{2}-3(4 r)^{2}$. We leave the details to the reader. 
Proof of Theorem 1.4. First insert the same Bailey pair from the previous proof with $a=1$ in (4). Then let $y \rightarrow \infty$, to obtain

$$
\begin{aligned}
1+(1-x) & \sum_{n=1}^{\infty}(q x)_{n-1} x^{-n}(-1)^{n} q^{n(n+1) / 2} \\
= & \frac{(q / x)_{\infty}}{(q)_{\infty}}\left(1+(1-x) \sum_{n=1}^{\infty} \frac{(x q)_{n-1} x^{-n}(-1)^{n} \alpha_{n} q^{n(n+1) / 2}}{(q / x)_{n}}\right),
\end{aligned}
$$

where we have used $\beta_{n}=1$. Now differentiate with respect to $x$, and set $x=1$ to get the following identity:

$$
\begin{aligned}
-\sum_{n=1}^{\infty}(q)_{n-1}(-1)^{n} q^{n(n+1) / 2}=\sum_{n=1}^{\infty} \frac{q^{n}}{\left(1-q^{n}\right)}-\sum_{n=1}^{\infty} \frac{q^{n^{2}+n}\left(1+q^{n}\right)}{\left(1-q^{n}\right)} \\
+\sum_{n=1}^{\infty}(-1)^{n} q^{n(5 n-1) / 2}\left(1+q^{n}\right) \sum_{j=-n+1}^{n-1}(-1)^{j} q^{-j(3 j+1) / 2}
\end{aligned}
$$

where we have used the fact that

$$
\begin{aligned}
-\sum_{n=1}^{\infty} \frac{\alpha_{n}(-1)^{n} q^{n(n+1) / 2}}{1-q^{n}} & =-\sum_{n=1}^{\infty} \frac{q^{n^{2}+n}\left(1+q^{n}\right)}{\left(1-q^{n}\right)} \\
+ & \sum_{n=1}^{\infty}(-1)^{n} q^{n(5 n-1) / 2}\left(1+q^{n}\right) \sum_{j=-n+1}^{n-1}(-1)^{j} q^{-j(3 j+1) / 2} .
\end{aligned}
$$

Now the left hand side of (8) generates the partitions counted by $L(n)$, weighted by -1 raised to 1 plus the number of parts. To see this, note the generating function of $L(n)$ is

$$
\sum_{n=0}^{\infty} L(n) q^{n}=\sum_{n=0}^{\infty}(-q)_{n} q^{(n+1)(n+2) / 2} .
$$

It is not difficult to see that the second sum on the right hand side of (8) is given by

$$
\sum_{n=1}^{\infty} \frac{q^{n^{2}+n}\left(1+q^{n}\right)}{\left(1-q^{n}\right)}=\sum_{n=1}^{\infty} \frac{q^{n^{2}}\left(1+q^{n}\right)}{\left(1-q^{n}\right)}-\sum_{n=1}^{\infty} q^{n^{2}}\left(1+q^{n}\right) .
$$

Thus, it can now be seen (by $[5$, p. 16, eq. (14.51)]) that we have the identity

$$
\begin{aligned}
& -\sum_{n=1}^{\infty}(q)_{n-1}(-1)^{n} q^{n(n+1) / 2} \\
= & \sum_{n=1}^{\infty} q^{n^{2}}\left(1+q^{n}\right)+\sum_{n=1}^{\infty}(-1)^{n} q^{n(5 n-1) / 2}\left(1+q^{n}\right) \sum_{j=-n+1}^{n-1}(-1)^{j} q^{-j(3 j+1) / 2},
\end{aligned}
$$


which reduces to

$$
\begin{aligned}
-\sum_{n=1}^{\infty}(q)_{n-1}(-1)^{n} & q^{n(n+1) / 2} \\
& =\sum_{n=1}^{\infty}(-1)^{n} q^{n(5 n-1) / 2}\left(1+q^{n}\right) \sum_{j=-n}^{n-1}(-1)^{j} q^{-j(3 j+1) / 2} .
\end{aligned}
$$

Paraphrasing this identity gives the theorem.

Proof of Theorem 1.5. This follows from Theorem 1.4, by using the fact that the equation

$$
n=r(5 r \pm 1) / 2-j(3 j+1) / 2
$$

is equivalent to

$$
360 n-6=(30 r \pm 3)^{2}-15(6 j+1)^{2} .
$$

The details are left to the reader.

3. Further results. The rank of a partition is defined as the largest part minus the number of parts. In this section, we notice that some results of [5] on the rank of a partition can be mixed with our Theorem 1.1 and Corollary 1.2 to give some interesting formulas for the functions $S_{3}(n)$ and $S_{1}(n)$. Fine's $\beta(n)$ function, the number of partitions of $n$ into odd parts without gaps, has the following generating function:

$$
\sum_{n=1}^{\infty} \beta(n) q^{n}=\sum_{n=1}^{\infty} \frac{q^{n^{2}}}{(1-q)\left(1-q^{3}\right) \cdots\left(1-q^{2 n-1}\right)},
$$

which is one of Ramanujan's third order mock theta functions alluded to in the introduction (see [5]). It is clear that

$$
S_{3}(n)+S_{1}(n)=\beta(n) .
$$

In keeping with the notation in [5], let $P_{r}(n ; Q)$ be the number of partitions of $n$ with rank $\equiv r(\bmod Q)$; then

$$
\begin{aligned}
\beta(2 n) & =P_{1}(2 n ; 4)-P_{2}(2 n ; 4), \\
\beta(2 n+1) & =P_{0}(2 n+1 ; 4)-P_{1}(2 n+1 ; 4),
\end{aligned}
$$

for any positive integer $n$. These two partition identities were obtained in [5], and will prove useful in establishing the following theorem:

Theorem 3.1. For $i=1$ or $i=2$ and any positive odd integer $n$, we have

$$
\begin{aligned}
& 2 S_{1}(n)=P_{0}(n ; 4)-P_{1}(n ; 4)+\frac{1}{2} s_{i}(1-24 n), \\
& 2 S_{3}(n)=P_{0}(n ; 4)-P_{1}(n ; 4)-\frac{1}{2} s_{i}(1-24 n) .
\end{aligned}
$$


Further, if $n$ is a positive even integer, then

$$
\begin{aligned}
& 2 S_{1}(n)=P_{1}(n ; 4)-P_{2}(n ; 4)-\frac{1}{2} s_{i}(1-24 n), \\
& 2 S_{3}(n)=P_{1}(n ; 4)-P_{2}(n ; 4)+\frac{1}{2} s_{i}(1-24 n) .
\end{aligned}
$$

Proof. By Theorem 1.1 and Corollary 1.2 we have

$$
(-1)^{n}\left(S_{3}(n)-S_{1}(n)\right)=\frac{1}{2} s_{i}(1-24 n)
$$

for $i=1$ or $i=2$. Taking $n$ to be an even positive integer, and adding $\beta(n)$ to (12), we obtain

$$
2 S_{3}(n)=P_{1}(n ; 4)-P_{2}(n ; 4)+\frac{1}{2} s_{i}(1-24 n) .
$$

Similarly, taking $n$ to be an odd positive integer and subtracting (12) from $\beta(n)$, we obtain

$$
2 S_{3}(n)=P_{0}(n ; 4)-P_{1}(n ; 4)-\frac{1}{2} s_{i}(1-24 n) .
$$

Similar computations for $S_{1}(n)$ give the other equations in the theorem.

4. Concluding remarks. There are a number of interesting points that should be mentioned from this study. First, it was pointed out by the referee that the function $f(n)$ is related to the factorization of $60 n-1$ in $\mathbb{Q}(\sqrt{3})$ and $\mathbb{Q}(\sqrt{5})$. In fact, it was further noted that this is another example of a $q$-series related to three different real quadratic fields (the first noted from [2]). Further research on this will be done in a subsequent study, as it would be much desired to find some further examples of $q$-series with this property.

Acknowledgments. The author would like to thank the referee for dramatically improving the original version of this paper, and pointing out Corollary 1.3 and its relevance to [2].

\section{References}

[1] G. E. Andrews, The fifth and seventh order mock theta functions, Trans. Amer. Math. Soc. 293 (1986), 113-134.

[2] G. E. Andrews, F. J. Dyson and D. Hickerson, Partitions and indefinite quadratic forms, Invent. Math. 91 (1988), 391-407.

[3] G. E. Andrews, J. Jiménez-Urroz and K. Ono, q-series identities and values of certain L-functions, Duke Math. J. 108 (2001), 395-419.

[4] W. N. Bailey, Identities of the Rogers-Ramanujan type, Proc. London Math. Soc. (2) 50 (1949), 1-10.

[5] N. J. Fine, Basic Hypergeometric Series and Applications, Math. Surveys Monogr. 27, Amer. Math. Soc., Providence, RI, 1988. 
[6] G. Gasper and M. Rahman, Basic Hypergeometric Series, Cambridge Univ. Press, Cambridge, 1990.

Alexander E. Patkowski

Department of Mathematics

University of Florida

Gainesville, FL 32611-8105, U.S.A.

E-mail: alexpatk@hotmail.com

Received June 13, 2008;

received in final form February 18, 2009 